University of Wollongong

Research Online

Faculty of Engineering and Information

Faculty of Engineering and Information

Sciences - Papers: Part B

Sciences

2017

\title{
Technical Note: Angular dependence of a 2D monolithic silicon diode array for small field dosimetry
}

\author{
Nauljun Stansook \\ University of Wollongong, ns938@uowmail.edu.au \\ Kananan Utitsarn \\ University of Wollongong, ku566@uowmail.edu.au \\ Marco Petasecca \\ University of Wollongong, marcop@uow.edu.au \\ Matthew Newall \\ University of Wollongong, mkn282@uowmail.edu.au \\ Mitchell Duncan \\ University of Wollongong, md640@uowmail.edu.au
}

See next page for additional authors

Follow this and additional works at: https://ro.uow.edu.au/eispapers1

Part of the Engineering Commons, and the Science and Technology Studies Commons

Research Online is the open access institutional repository for the University of Wollongong. For further information contact the UOW Library: research-pubs@uow.edu.au 


\section{Technical Note: Angular dependence of a 2D monolithic silicon diode array for small field dosimetry}

\section{Abstract \\ Purpose}

This study aims to investigate the 2D monolithic silicon diode array size of $52 \times 52 \mathrm{~mm}^{2}$ (MP512) angular response. An angular correction method has been developed that improves the accuracy of dose measurement in a small field.

\section{Methods}

The MP512 was placed at the center of a cylindrical phantom, irradiated using $6 \mathrm{MV}$ and $10 \mathrm{MV}$ photons and incrementing the incidence of the beam angle in $15^{\circ}$ steps from $0^{\circ}$ to $180^{\circ}$, and then in $1^{\circ}$ steps between $85^{\circ}$ and $95^{\circ}$. The MP512 response was characterized for square field sizes varying between $1 \times$ $1 \mathrm{~cm}^{2}$ and $10 \times 10 \mathrm{~cm}^{2}$. The angular correction factor was obtained as the ratio of MP512 response to EBT3 film measured doses as a function of the incidence angle $(\theta)$ and was normalized at $0^{\circ}$ incidence angle. Beam profiles of the corrected MP512 responses were compared with the EBT3 responses to verify the effectiveness of the method adopted.

\section{Results}

The intrinsic angular dependence of the MP512 shows maximum relative deviation from the response normalized to $0^{\circ}$ of $18.5 \pm 0.5 \%$ and $15.5 \pm 0.5 \%$ for $6 \mathrm{MV}$ and $10 \mathrm{MV}$, respectively, demonstrating that the angular response is sensitive to the energy. In contrast, the variation of angular response is less affected by field size. Comparison of cross-plane profiles measured by the corrected MP512 and EBT3 shows an agreement within $\pm 2 \%$ for all field sizes when the beams irradiated the array at $0^{\circ}, 45^{\circ}, 135^{\circ}$, and $180^{\circ}$ angles of incidence from the normal to the detector plane. At $90^{\circ}$ incidence, corresponding to a depth dose measurement, up to a $6 \%$ discrepancy was observed for a $1 \times 1 \mathrm{~cm}^{2}$ field of $6 \mathrm{MV}$.

\section{Conclusion}

An angular correction factor can be adopted for small field sizes. Measurements discrepancies could be encountered when irradiating with very small fields parallel to the detector plane. Using this approach, the MP512 is shown to be a suitable detector for 2D dose mapping of small field size photon beams.

\section{Disciplines}

Engineering | Science and Technology Studies

\section{Publication Details}

Stansook, N., Utitsarn, K., Petasecca, M., Newall, M. K., Duncan, M., Nitschke, K., Carolan, M., Metcalfe, P., Lerch, M. L. F., Perevertaylo, V. L., Tome, W. A. \& Rozenfeld, A. B. (2017). Technical Note: Angular dependence of a 2D monolithic silicon diode array for small field dosimetry. Medical Physics, 44 (8), 4313-4321.

\section{Authors}

Nauljun Stansook, Kananan Utitsarn, Marco Petasecca, Matthew Newall, Mitchell Duncan, Kym N. Nitschke, Martin G. Carolan, Peter E. Metcalfe, Michael L. F Lerch, Vladimir Perevertaylo, Wolfgang Tome, and Anatoly B. Rosenfeld 
Technical Note: Angular dependence of a 2D monolithic silicon diode array for small field dosimetry

Nauljun Stansook ${ }^{1,2}$, Kananan Utitsarn ${ }^{1}$, Marco Petasecca ${ }^{1,3}$, Matthew K. Newall ${ }^{1}$, Mitchell

Duncan $^{1}$, Kym Nitschke ${ }^{3}$, Martin Carolan ${ }^{1,3,4}$, Peter Metcalfe ${ }^{1}$, Michael L.F. Lerch ${ }^{1,3}$, Vladimir

5 L. Perevertaylo ${ }^{5}$, Wolfgang A. Tomé ${ }^{1,6}$, Anatoly B. Rosenfeld ${ }^{1,3,(a)}$

${ }^{1}$ Centre for Medical Radiation Physics, University of Wollongong, NSW 2500, Australia.

${ }^{2}$ Division of Radiation Oncology, Department of Radiology, Faculty of Medicine, Ramathibodi Hospital, Mahidol University, Bangkok 10400, Thailand

${ }^{3}$ Illawarra Heath Medical Research Institute, Wollongong, NSW 2522, Australia.

$10{ }^{4}$ Illawarra Cancer Care Centre, Wollongong Hospital, Wollongong, NSW 2500, Australia.

${ }^{5}$ SPA BIT, Kiev 02232, Ukraine.

${ }^{6}$ Department of Radiation Oncology, Albert Einstein College of Medicine, NY 10461, USA.

a) Author to send correspondence to. Electronic mail: anatoly@uow.edu.au. 


\begin{abstract}
:
Purpose: This study aims to investigate the $2 \mathrm{D}$ monolithic silicon diode array size of $52 \times 52$ $\mathrm{mm}^{2}$ (MP512) angular response. An angular correction method has been developed that improves the accuracy of dose measurement in a small field.
\end{abstract}

20 Methods: The MP512 was placed at the center of a cylindrical phantom, irradiated using 6 MV and $10 \mathrm{MV}$ photons and incrementing the incidence of the beam angle in $15^{\circ}$ steps from $0^{\circ}$ to $180^{\circ}$, and then in $1^{\circ}$ steps between $85^{\circ}$ to $95^{\circ}$. The MP512 response was characterized for square field sizes varying between $1 \times 1 \mathrm{~cm}^{2}$ to $10 \times 10 \mathrm{~cm}^{2}$. The angular correction factor was obtained as the ratio of MP512 response to EBT3 film measured doses as a function of the incidence angle $(\Theta)$ and was normalized at $0^{\circ}$ incidence angle. Beam profiles of the corrected MP512 responses were compared with the EBT3 responses to verify the effectiveness of the method adopted.

Results: The intrinsic angular dependence of the MP512 shows maximum relative deviation from the response normalized to $0^{\circ}$ of $18.5 \pm 0.5 \%$ and $15.5 \pm 0.5 \%$ for $6 \mathrm{MV}$ and $10 \mathrm{MV}$, respectively, demonstrating that the angular response is sensitive to the energy. In contrast, the variation of angular response is less affected by field size. Comparison of cross-plane profiles measured by the corrected MP512 responses and EBT3 responses shows an agreement within $\pm 2 \%$ for all field sizes when the beam irradiated the array at $0^{\circ}, 45^{\circ}, 135^{\circ}$, and $180^{\circ}$ angles of incidence from the normal to the detector plane. At $90^{\circ}$ incidence, corresponding to a depth dose measurement, up to a $6 \%$ discrepancy was observed for a $1 \times 1 \mathrm{~cm}^{2}$ field of $6 \mathrm{MV}$.

35 Conclusion: An angular correction factor can be adopted for small field sizes. Measurements discrepancies could be encountered when irradiating with very small fields parallel to the detector plane. Using this approach, the MP512 is shown to be a suitable detector for 2D dose mapping of small field size photon beams.

Key words: monolithic 2D detector, stereotactic radiotherapy, small field dosimetry, quality assurance, silicon diode 


\section{INTRODUCTION}

Stereotactic radiosurgery (SRS), stereotactic body radiotherapy (SBRT) and stereotactic ablative radiotherapy (SABR) are increasingly being employed in radiation therapy because of their superior conformity and radiobiological effectiveness over conventional photon therapies. ${ }^{1}$ In these forms of therapy, high doses per fraction are delivered to small targets using stereotactic localization techniques while limiting dose to normal tissue and critical organs ${ }^{2,3}$. The use of small fields combined with intensity-modulated delivery creates a challenging scenario for accurate dosimetry. The most critical issues in small field dosimetry are a lack of electronic equilibrium resulting from the field dimension being less than the secondary electron lateral range, and the possible partial obstruction of the source by collimators. The secondary electron lateral range is also energy dependent and therefore, its impact on penumbra and effective beam size also depends on the density of the medium. ${ }^{4}$ A suitable quality assurance tool for small field dosimetry requires high spatial resolution detectors, tissue equivalence, and energy and dose rate independence to achieve sufficient accuracy. The packaging of the detector should also only minimally perturb the radiation field. ${ }^{5}$

Recently, 2D detector arrays based on either diodes or ionization chambers have been used as verification tools due to their convenient real-time dose measurement capabilities. The Centre for Medical Radiation Physics (CMRP) has developed a monolithic silicon diode array named Magic Plate-512 (MP512) with pixel pitch of $2 \mathrm{~mm}$ for dosimetry verification in stereotactic RT, including real time motion adaptive radiotherapy. In terms of beam profile and penumbra measurement, the MP512 has proved to be a suitable dosimeter for external beam radiotherapy and small field dosimetry for field sizes down to $1 \mathrm{x} 1 \mathrm{~cm}^{2} \cdot{ }^{6,7}$ However, the major drawback of monolithic silicon diode arrays is angular dependence.

The angular dependence of diodes and diode arrays have been reported in several recent studies. $^{8-10}$ Anisotropy in materials surrounding the detector active volume and detector assembly are the two main factors that affect the angular response of the detector, since the different materials surrounding the active volume generate a varying secondary electron spectrum depending on irradiation angle. ${ }^{9,11-14}$ This effect produces an angular dependence and 
limits the accuracy of verification plans for arc radiotherapy delivery. Several groups have found that for dosimetry systems exhibiting angular dependence, mitigation of it using an angular dependence correction methodology allows their use for plan verification with acceptable accuracy. ${ }^{15-20}$ The aims of this study are: 1) to investigate the characteristics of the MP512 in terms of its intrinsic directional dependence, including the effects of photon energy and field size on its angular response; and 2) to develop an angular correction factor procedure to improve the accuracy of the measured dose map in a specific plane after full plan delivery for arc radiotherapy using small fields as employed in SBRT and SRS.

\section{MATERIALS AND METHODS}

\section{A. MagicPlate-512 monolithic detector array and data acquisition system (DAQ)}

The MP512 is a monolithic silicon detector array of 512 square diodes distributed over an area of $52 \times 52 \mathrm{~mm}^{2}$. Each pixel has an active area of $0.5 \times 0.5 \mathrm{~mm}^{2}$ and a pitch of $2 \mathrm{~mm}$. The detector array is fabricated by ion implantation on a $470 \mu \mathrm{m}$ thick p-type silicon substrate. The MP512 is wire bonded to a $500 \mu \mathrm{m}$ thick fiber glass printed circuit board (PCB) with plugs for connection to a readout DAQ system. The detector and the wire bonding are covered by a thin layer of protective epoxy for mechanical robustness. The MP512 detector array was embedded in between two $5 \mathrm{~mm}$ thick PMMA slabs with an air gap of $1 \mathrm{~mm}$ between the plastic cover and the silicon entrance surface. This packaging arrangement (cf. Fig. 1) is necessary for mechanical protection of the silicon detector and to optimize the detector response to normally incident small radiation fields. ${ }^{6,21,22}$

The MP512 data acquisition system (DAQ) is based on eight modules of 64-channel analog electrometers. ${ }^{6,23,24}$ Each chip is interfaced to a quad analogue-to-digital converter (ADC). The ADC output is synchronized and channel de-randomized by a field programmable gate array (FPGA), which also manages the synchronization with the sync pulse of the Linac to acquire the detector current. The data was transferred from FPGA via USB2.0 to a host computer on which the in-house developed program interface operates. The MP512 array is operated in passive mode, where no bias voltage is applied to the diodes. 


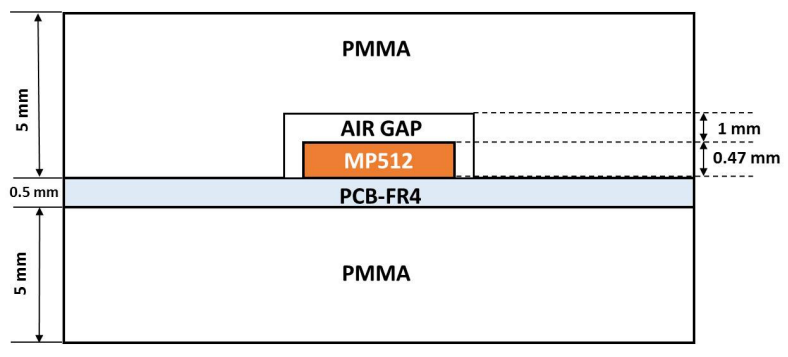

Figure 1. The MP512 schematic diagram of cross-section of the detector packaging (not to scale)

\section{B. Angular response}

Angular response measurements were performed using $6 \mathrm{MV}$ and $10 \mathrm{MV}$ photon beams generated by a Varian Clinac iX (Varian Medical System, Palo Alto, CA) equipped with a 120leaf Millennium multi-leaf collimator system. The MP512 was inserted into the PMMA cylindrical phantom having a diameter of $30 \mathrm{~cm}$ and a length of $40 \mathrm{~cm}$ (cf. Fig.2a), with the central pixel aligned with the machine's isocenter. To avoid couch interference, the detector was set in a vertical position with the detector surface perpendicular to the incident beam. The 0 degree angle is identified with the gantry in horizontal position (cf. Fig. 2b). Square field sizes of $1 \times 1 \mathrm{~cm}^{2}, 2 \times 2 \mathrm{~cm}^{2}, 3 \times 3 \mathrm{~cm}^{2}, 4 \times 4 \mathrm{~cm}^{2}$ and $10 \times 10 \mathrm{~cm}^{2}$ were adopted to irradiate the detector, rotating the gantry in a clockwise direction at $15^{\circ}$ increments from incidence beam angle $0^{\circ}$ to $+180^{\circ}$. For the beam with field size $10 \times 10 \mathrm{~cm}^{2}$, the gantry was rotated in $1^{\circ}$ increments for incidence beam angles between $85^{\circ}$ to $95^{\circ}$ for a finer characterization of the detector around the expected maximum response variation. The MP512 was irradiated five times using $100 \mathrm{MU}$ for each angular position at a dose rate of $600 \mathrm{MU} / \mathrm{min}$. The angular response was defined as the ratio of the detector response at a given irradiation angle normalized to the response at $0^{\circ}$ incidence angle. As the data in this study was not normally distributed, and the sample size per group less than the criteria $(\mathrm{n}<15)$, nonparametric statistical analysis was used. A Kruskal-Wallis test was carried out to compare the effect of field size on angular response variation for the same photon energy, while a Mann-Whitney $\mathrm{U}$ test was conducted to compare the angular response between the two photon energies of $6 \mathrm{MV}$ and $10 \mathrm{MV}$, respectively. 


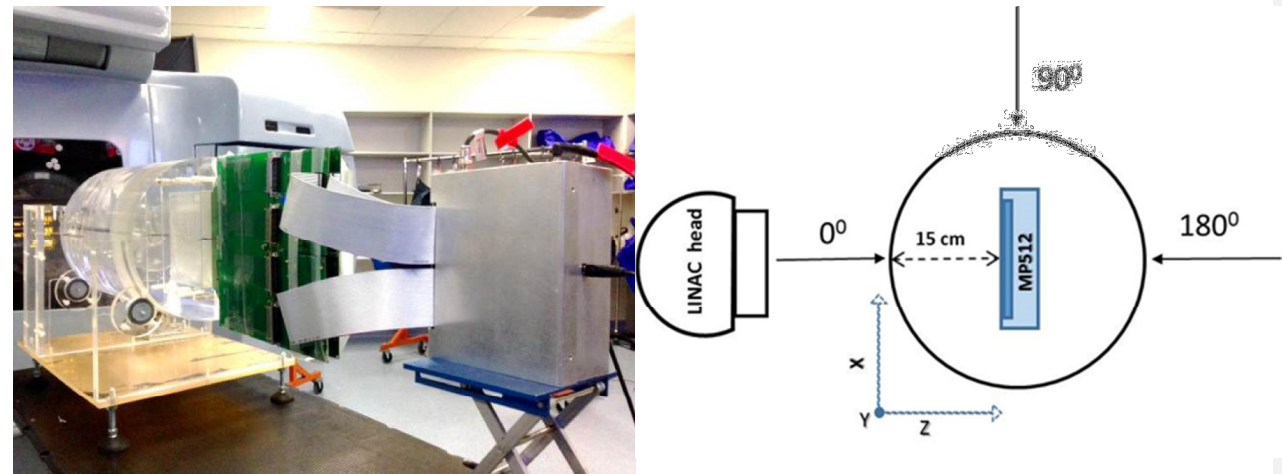

(a)

(b)

Figure 2. The experiment setup; (a) The MP512 placed inside the PMMA cylindrical phantom, (b) Schematic diagram of directional measurement (not to scale).

\section{C. Gafchromic EBT3 film and correction factor calculation}

Gafchromic EBT3 film was used as the reference dosimeter to evaluate and correct the angular responses of MP512, assuming that the film response does not have any appreciable angular dependence. EBT3 film has been designed to minimize its angular response by using two identical active layers stacked with two matte polyester layers for protection from mechanical damage. ${ }^{25}$ Normalization of the MP512 detector response to the EBT3 film response helps to minimize the effects of mechanical tolerances of rotation of the Linac gantry around the phantom, and effects of non-homogeneity of the phantom.

The EBT3 films were cut into $7.0 \times 7.4 \mathrm{~cm}^{2}$ patches to fit the PMMA holder used for MP512 packaging. The films were irradiated using $6 \mathrm{MV}$ and $10 \mathrm{MV}$ beams with a $10 \times 10 \mathrm{~cm}^{2}$ open field at incidence beam angles of $0^{\circ}, 45^{\circ}, 90^{\circ}, 135^{\circ}$, and $180^{\circ}$ in the same position as the detector array. The set of films were scanned six times at the same position in the center of a Microtrex ScanMaker i800 flatbed scanner with 48-bit of color depth and spatial resolution of 72 dpi (equivalent to a pixel size of $350 \mu \mathrm{m}$ ). The last three scans were used to perform the analysis to ensure thermal stability of the scanner and inter-scanning consistency. ${ }^{26}$ The red channel was used for data analysis using ImageJ V1.48 (National Institute of Health, USA) and MATLAB 2014b (Math Works Inc., Natick, MA). 
The response of the film at the required $15^{\circ}$ angular increments for determination of an angular correction of MP512, was obtained by interpolating the data obtained at $0^{\circ}, 45^{\circ}, 90^{\circ}$, $135^{\circ}$, and $180^{\circ}$. The data collected using the MP512 and EBT3 film for an open $10 \times 10 \mathrm{~cm}^{2}$ field was used to determine the correction factor for each detector pixel. Increasing the field size above $10 \times 10 \mathrm{~cm}^{2}$ for angular calibration would not typically be measured using a $52 \times 52 \mathrm{~mm}^{2}$ detector array that was designed for stereotactic fields. The calibration factor $a_{i j}$ expressed in counts/cGy for each pixel $(\mathrm{i}, \mathrm{j})$, was calculated from the ratio of MP512 response to dose measured by the EBT3 film as a function of the gantry angle $\theta$ (cf. Equation (1)).

$$
a_{i j}(\theta)=\frac{M P_{i j}(\theta)}{E B T 3_{i j}(\theta)}
$$

where, $i$ is the row index (along the $x$-axis, i.e. axis perpendicular to the phantom rotation) and $j$ is the column index of pixels (along the $y$-direction, i.e. parallel to the axis of rotation).

The angular response calibration tensor of the MP512 $\left(C_{i j}(\theta)\right)$ was calculated by dividing the calibration factor at an arbitrary gantry angle $\theta$ by the calibration factor at gantry angle zero (cf. Equation (2)).

$$
C_{i j}(\theta)=\frac{a_{i j}(\theta)}{a_{i j}(0)}
$$

\section{D. Verification of angular dependence correction factor}

To verify the constancy of the angular response correction factor of the MP512, experiments were performed using small static beam irradiation and compared with those measured using EBT3 films set up in the same orientation (cf. Fig. 2a). The MP512 was irradiated using open field sizes of $1 \times 1 \mathrm{~cm}^{2}, 2 \times 2 \mathrm{~cm}^{2}, 3 \times 3 \mathrm{~cm}^{2}$ and $4 \times 4 \mathrm{~cm}^{2}$ at incidence gantry angles of $0^{\circ}, 45^{\circ}, 90^{\circ}$, $135^{\circ}$ and $180^{\circ}$ for both $6 \mathrm{MV}$ and $10 \mathrm{MV}$ photons. The dose map measured by MP512 ( $\left.M P_{i j}^{\text {corrected }}(\theta)\right)$ corrected for angular dependence was obtained by dividing the intrinsic detector response $\left(M P_{i j}^{\prime}(\theta)\right)$ by the calibration tensor $C_{i j}(\theta)$ for the $10 \times 10 \mathrm{~cm}^{2}$ field (cf. Equation (3)).

$$
M P_{i j}^{\text {corrected }}=\frac{M P_{i j}^{\prime}(\theta)}{C_{i j}(\theta)}
$$

\section{RESULTS}




\section{A. Angular response}

Figure 3 shows the intrinsic detector response between $0^{\circ}$ and $180^{\circ}$ as a function of the incidence angle collected from the four central pixels for various field sizes. The error bars shown are $95 \%$ confidence intervals with a maximum variation of $\pm 0.2 \%$. The detector response decreases as the incidence beam angle increases, giving an average response change per degree of $0.18 \pm 0.03 \%$. For all studied field sizes and photon energies, the relative angular response of the MP512 is similar, with a minimum response of $18.5 \pm 0.5 \%$ for $6 \mathrm{MV}$ and $15.5 \pm 0.5 \%$ for 10 MV achieved at incidence angles between $90^{\circ}$ and $95^{\circ}$. As the incidence angle increases from $90^{\circ}$ to $180^{\circ}$, the detector response recovers asymmetrically and falls below the response at $0^{\circ}$ degrees by $14.3 \pm 0.6 \%$ for $6 \mathrm{MV}$ and $9.4 \pm 1.8 \%$ for $10 \mathrm{MV}$ (cf. Fig. 3). There was no significant difference in the relative angular response in between the different field sizes, yielding a value of $\mathrm{p}=0.9985$ for $6 \mathrm{MV}$ and $\mathrm{p}=0.5359$ for $10 \mathrm{MV}$. However, for a $10 \mathrm{MV}$ photon beam at incidence angles between $135^{\circ}$ to $180^{\circ}$, the relative response differences between a field size of $1 \times 1 \mathrm{~cm}^{2}$ and a field size of $10 \times 10 \mathrm{~cm}^{2}$ were approximately $3 \%$. Figure 4 shows the intrinsic detector response for an open field size of $10 \times 10 \mathrm{~cm}^{2}$ between $0^{\circ}$ and $180^{\circ}$ as a function of the beam incidence angle collected from the four central pixels for $6 \mathrm{MV}$ and $10 \mathrm{MV}$ photon beam energies. The angular response for the higher energy $10 \mathrm{MV}$ photon beams shows a smaller variation than for $6 \mathrm{MV}$ photon beams proving that there is a significant difference $(\mathrm{p}=0.04)$ in the angular dependence of monolithic arrays as a function the beam energy.
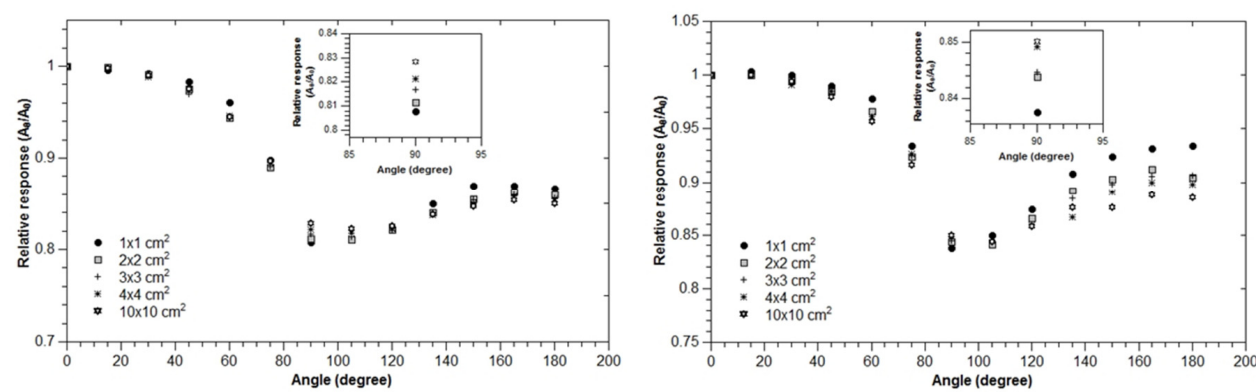

(a)

(b)

Figure 3. Angular response of the averaged four central pixels of the MP512 detector array is shown as a function of incidence beam angle for (a) $6 \mathrm{MV}$ and (b) $10 \mathrm{MV}$ photons. 


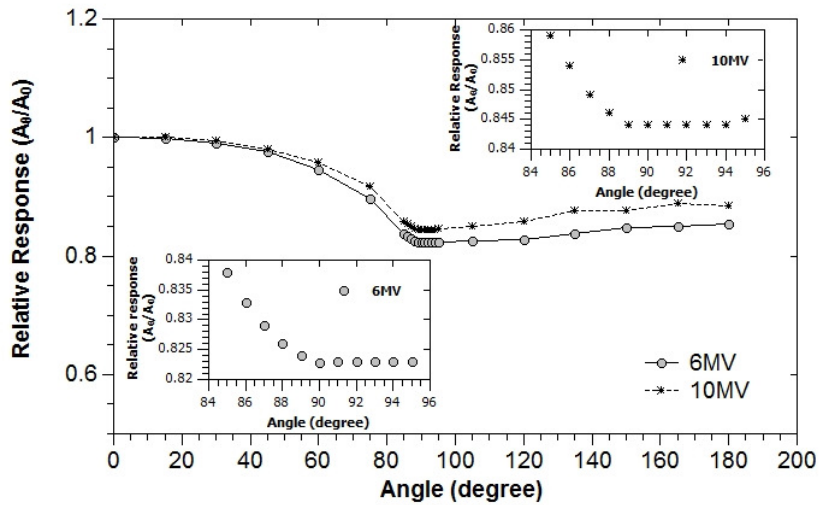

Figure 4. Angular response of the averaged four central pixels of the MP512 detector array is shown as a function of incidence beam angle for an open field size of 10x10 $\mathrm{cm}^{2}$ for $6 \mathrm{MV}$ and $10 \mathrm{MV}$ photons.

\section{B. Validating the angular response correction factor for small fields}

The beam profiles for field sizes from $1 \times 1$ to $4 \times 4 \mathrm{~cm}^{2}$ measured using the MP512 at an incidence angle of 0 degrees, and compared to EBT3 films, show an agreement in terms of the full width of half maximum (FWHM) and penumbra (20-80\%) for 6 MV and 10 MV photon beams within $\pm 1 \%$ and $1 \mathrm{~mm}$, respectively. When uncorrected for angular dependence, the MP512 response to beams at incidence angles other than zero degrees shows a dose profile distorted, and with considerable attenuation, when compared to EBT3 film. The profiles from $1 \mathrm{x} 1$ to $4 \mathrm{x} 4 \mathrm{~cm}^{2}$ measured with the MP512 detector are substantially improved after correction, and match to EBT3 dose profiles for all angles to within $\pm 2 \%$ (cf. Fig. 5 to 8 ) for both 6 and 10 MV photon beams. However, for $6 \mathrm{MV}$ photons and a $1 \mathrm{x} 1 \mathrm{~cm}^{2}$ field size, the cross-plane depthdose profiles corresponding to a gantry angle of $90^{\circ}$ showed a discrepancy of approximately $6 \%$ in comparison to EBT3 film after angular correction (cf. Fig. 7c). 


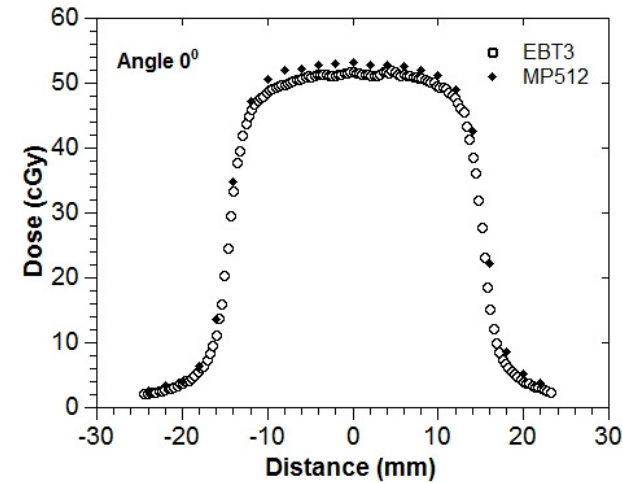

(a)

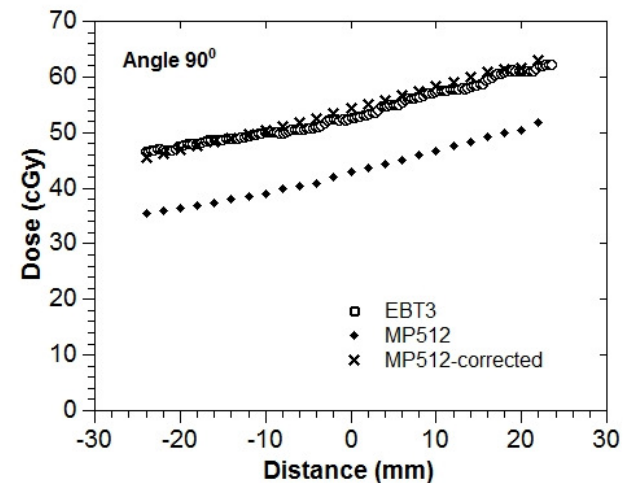

(b)

Figure 5. Comparison of dose profiles measured with EBT3 films and reconstructed from MP512 without and with angular correction for a $3 \times 3 \mathrm{~cm}^{2}$ open field for $6 \mathrm{MV}$ photons for different beam incidence angles; (a) $0^{\circ}$ and (b) $90^{\circ}$.

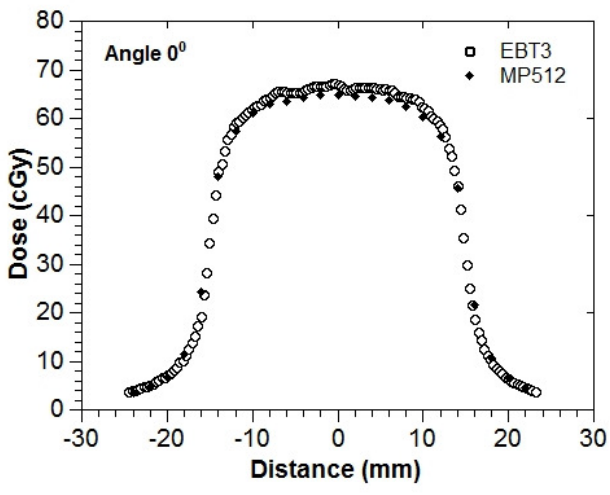

(a)

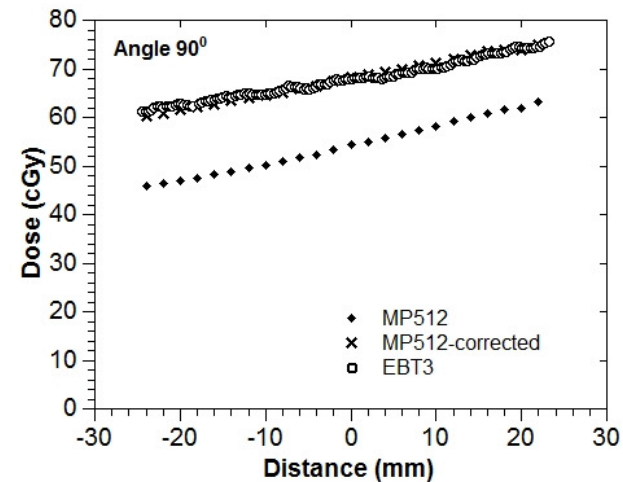

(b)

Figure 6. Comparison of dose profiles measured with EBT3 films and reconstructed from MP512 without and with angular correction for a $3 \times 3 \mathrm{~cm}^{2}$ open field for $10 \mathrm{MV}$ photons for different beam incidence angles; (a) $0^{\circ}$ and (b) $90^{\circ}$. 

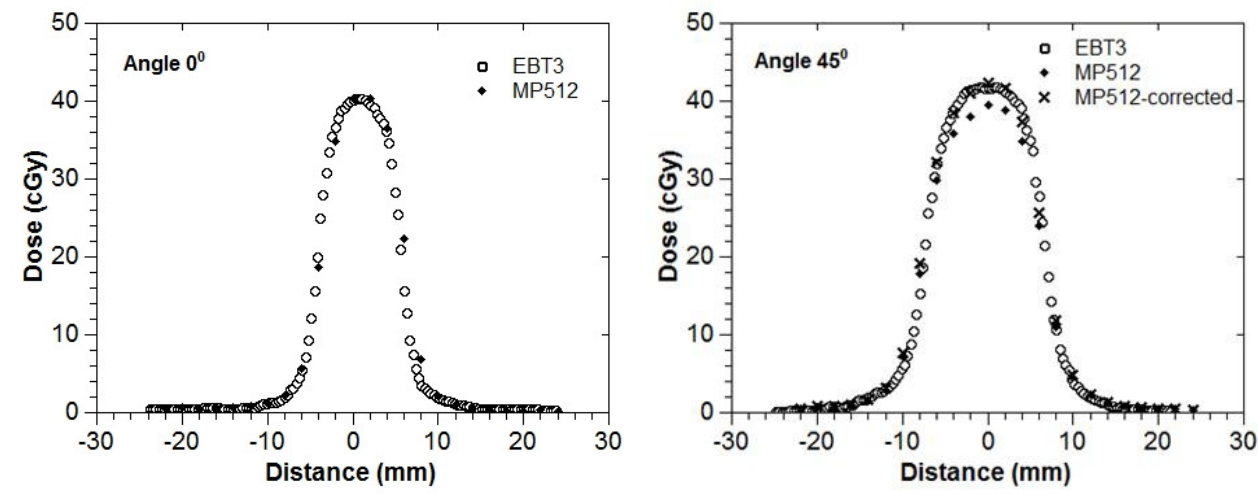

(a)

(b)
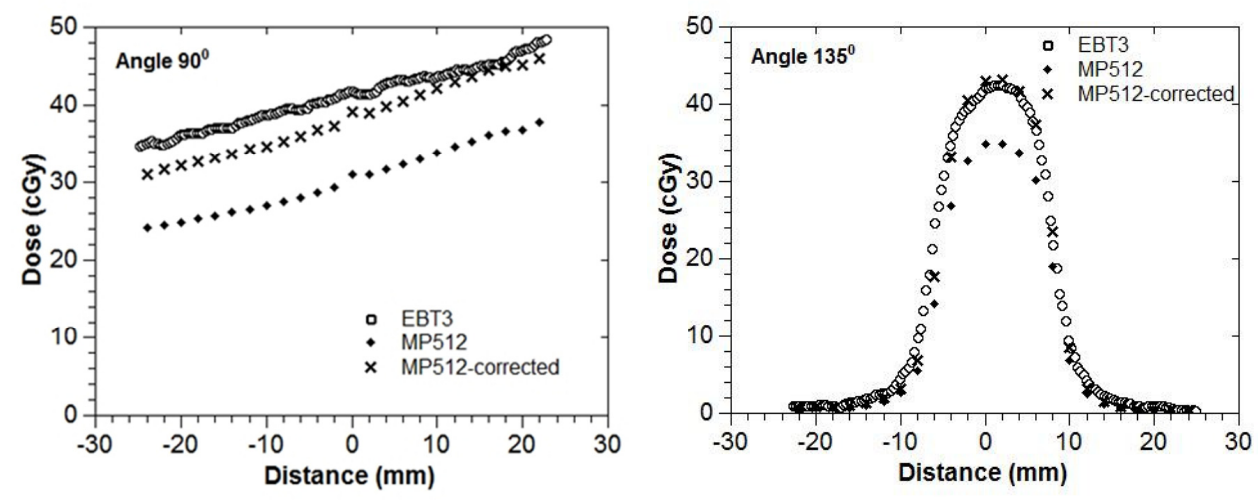

(c)

(d)

Figure 7. Comparison of dose profiles measured with EBT3 films and reconstructed from MP512 without and with angular correction for a $1 \times 1 \mathrm{~cm}^{2}$ open field for $6 \mathrm{MV}$ photons for different beam incidence angles; (a) $0^{\circ}$, (b) $45^{\circ}$, (c) $90^{\circ}$ and (d) $135^{\circ}$. 

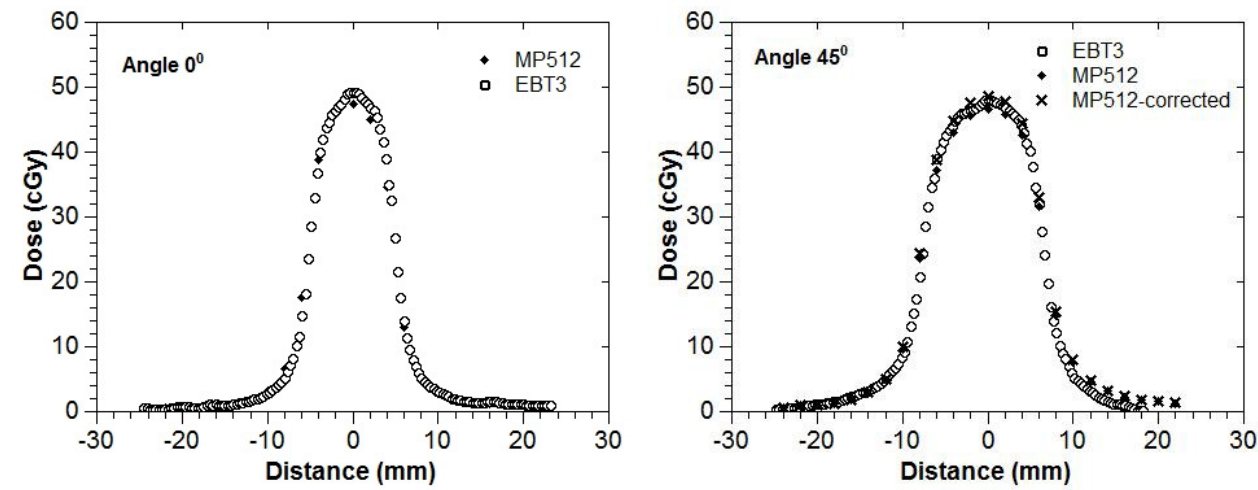

(a)

(b)
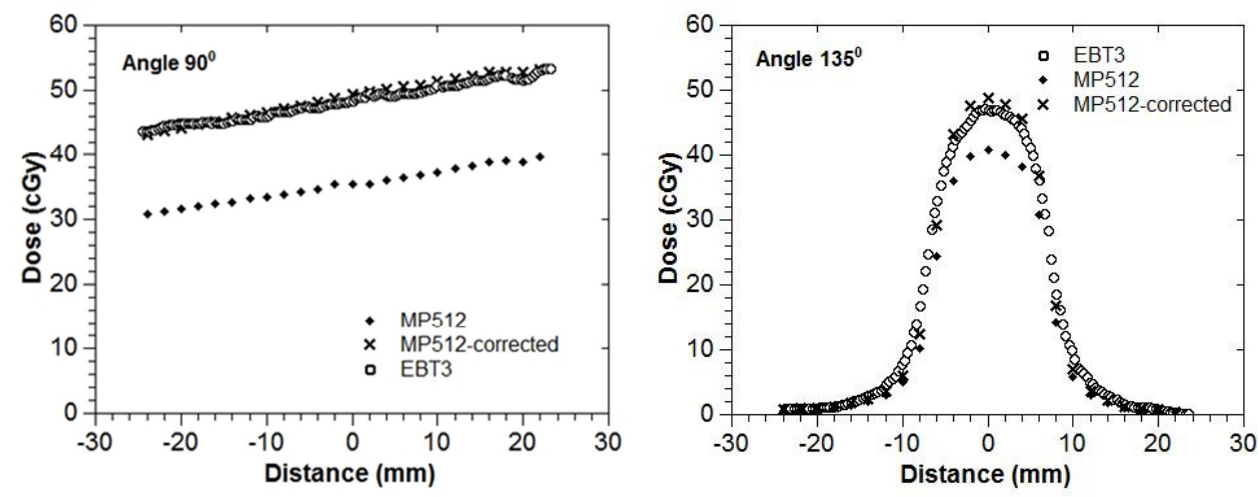

(c)

(d)

Figure 8. Comparison of dose profiles measured with EBT3 films and reconstructed from MP512 without and with angular correction for a $1 \times 1 \mathrm{~cm}^{2}$ open field for $10 \mathrm{MV}$ photons for different beam incidence angles; (a) $0^{\circ}$, (b) $45^{\circ}$, (c) $90^{\circ}$ and (d) $135^{\circ}$.

\section{DISCUSSION}

We found that the relative angular dose response of the MP512 decreases with increasing of the beam incidence angle by up to $18.5 \pm 0.5 \%$ for $6 \mathrm{MV}$ and $15.5 \pm 0.5 \%$ for $10 \mathrm{MV}$ in comparison to normal beam incidence $(0$ degree $)$ with the minimum achieved between $90^{\circ}$ and $95^{\circ}$. The minimum of the relative detector response was observed when radiation beam was parallel to the silicon plane of the MP512. This effect can be explained by the higher attenuation of the portion of beam passing through $26 \mathrm{~mm}$ length of the $0.47 \mathrm{~mm}$ thick silicon substrate, as well as the perturbation of the lateral equilibrium and scattered secondary electrons by the silicon substrate 
and the air gap surrounding the silicon crystal. It was observed that for $1 \mathrm{x} 1 \mathrm{~cm}^{2}$ field of $10 \mathrm{MV}$ beams, the relative angular response differed by more than $3 \%$ in the angular interval $135^{\circ}-180^{\circ}$ in comparison with the response for $10 \times 10 \mathrm{~cm}^{2}$ field, while for all other fields agreement is good. The proportion of this field in practical delivery of arc therapy will be small in terms of contribution to the total dose measured by MP512, and essentially will not influence dose reconstruction to the total dose measured by MP512. This will be demonstrated in the next paper. Further analysis of this phenomena is required. While the MP512 is a large monolithic 2D array detector, its angular response variation is similar to the angular response of single diodes found in other studies. ${ }^{9,11,27}$ The reason for the asymmetric directional dependence of the MP512 can be attributed to the intrinsic anisotropic configuration of the MP512 silicon detector assembly where each pixel of MP512 is surrounded laterally by extracameral silicon and high atomic number materials such as the compounds used for fabrication of the printed fiberglass circuit board adopted to connect the detector to the electronic interface. The packaging solution adopted leads to beam angle-dependent attenuation differences of the secondary electrons, and hence results in the angular dependent sensitivity of the detector pixels. In addition, the silicon surrounding the detector pixels sensitive volume has a density higher than water, which leads to the production of secondary electrons with an energy distribution that is different to that generated in water, affecting the dose measured by the detector pixels under different beam angles. ${ }^{14,28-30}$ For a $10 \mathrm{MV}$ photon beam, the secondary electrons have higher energy in comparison to those generated from a $6 \mathrm{MV}$ photon beam leading to a less pronounced angular response of the MP512. However, for potentially evaluating, the Monte Carlo simulations is a powerful tool allowing to understand the effect of extracameral volume around detector pixels and the detector packaging on angular dependence of the detector and further it optimization.

The angular correction tensor is calculated by normalizing the EBT3 film dose map and the MP512 response for a photon beam with field size of $10 \times 10 \mathrm{~cm}^{2}$. This methodology yielded a cross-plane profile agreement to within 2\% between the corrected MP512 dose response and EBT3 film dose measurement for all studied field sizes for both $6 \mathrm{MV}$ and $10 \mathrm{MV}$ photons, except for the $1 \times 1 \mathrm{~cm}^{2}$ for $6 \mathrm{MV}$ for which the agreement was within $6 \%$ at an incidence angle 
of $90^{\circ}$ corresponding to a depth dose measurement. The observed discrepancy can be explained by the fact that for $1 \times 1 \mathrm{~cm}^{2}$ field in comparison with a $10 \times 10 \mathrm{~cm}^{2}$ field, the partial fraction of photons attenuated by the $0.47 \mathrm{~mm}$ thick and $52 \mathrm{~mm}$ long silicon substrate has a much larger influence on the detector pixel response embedded in silicon with depth. While for a $10 \times 10 \mathrm{~cm}^{2}$ field, the detector pixel response with depth is driven mostly by secondary electrons scattered from the PMMA to the silicon. Also, the airgap between the silicon detector and the PMMA cover is part of the extra-cameral material and allows a non-negligible fraction of the secondary electrons in a $1 \times 1 \mathrm{~cm}^{2}$ field to travel along the silicon substrate without interacting with the detector perturbing the energy deposition distribution in respect to a field size of $10 \times 10 \mathrm{~cm}^{2}$. In the case of the $1 \times 1 \mathrm{~cm}^{2}$ field and $10 \mathrm{MV}$ photon beam, this effect is less pronounced due to higher energy of the secondary electrons that leads to a smaller beam perturbation and asymmetry created by the PCB and silicon extracameral material.

\section{CONCLUSION}

This study aims to characterize the intrinsic angular response of the monolithic silicon detector array MP512 for different field sizes and photon energies. The angular response of the MP512 is found to be independent of field sizes. There are significant differences of MP512 angular response between 6 and 10MV photon energies at a fixed beam size of $10 \mathrm{x} 10 \mathrm{~cm}^{2}$. The packaging and the intrinsic asymmetry of monolithic silicon detectors are the major elements affecting the angular dependence of the MP512. An angular correction factor obtained for a $10 \times 10 \mathrm{~cm}^{2}$ field size can also be utilized for smaller field sizes, however, for 6MV photon field size of $1 \times 1 \mathrm{~cm}^{2}$, a different correction factor is required when the beam is directed along the silicon detector (i.e. at 90 or 270 degrees) and will require the further investigation.

\section{ACKNOWLEDGMENTS}

Nauljun Stansook was supported by faculty of Medicine Ramathibodi Hospital, Mahidol University, Thailand. The authors would like to acknowledge the National Health and Medical

Research Council of Australia that funded this project by the Project Grant No. GNT1030159.

\section{CONFLICT OF INTEREST}

The authors have no relevant conflicts of interest to disclose. 


\section{REFERENCES}

1. Timmerman RD, Kavanagh BD. Stereotactic body radiation therapy. Curr Probl Cancer. 2005;29(3):120-157. doi:10.1016/j.currproblcancer.2005.05.001.

2. Potters L, Steinberg M, Rose C, et al. American Society for Therapeutic Radiology and Oncology and American College of Radiology practice guideline for the performance of stereotactic body radiation therapy. Int J Radiat Oncol Biol Phys. 2004;60(4):1026-1032. doi:10.1016/j.ijrobp.2004.07.701.

3. Lo SS, Chang EL, Yamada Y, Sloan AE, Suh JH, Mendel E. Stereotactic radiosurgery and radiation therapy for spinal tumors. Expert Rev Neurother. 2007;7(1):85-93. doi:10.1586/14737175.7.1.85.

4. Papież L, Timmerman R, Desrosiers C, Randall M. Extracranial Stereotactic Radioablation Physical Principles. Acta Oncol (Madr). 2003;42(8):882-894. doi:10.1080/02841860310013490.

5. Wuerfel JU. Dose measurements in small fields. Med Phys Int. 2013;1(1):81-90.

6. Aldosari A H, Petasecca M, Espinoza A, et al. A two dimensional silicon detectors array for quality assurance in stereotactic radiotherapy: MagicPlate-512. Med Phys. 2014;091707(41):091707. doi:10.1118/1.4892384.

280 7. Petasecca M, Newall MK, Booth JT, et al. MagicPlate-512: A 2D silicon detector array for quality assurance of stereotactic motion adaptive radiotherapy. Med Phys. 2015;42(6):2992-3004. doi:10.1118/1.4921126.

8. Lee PC, Sawicka JM, Glasgow GP. Patient Dosimetry Quality Assurance Program with a Commercial Diode System. Int. J. Radiat. Oncol., Biol., Phys. 1994;29(5):1175-1182.

285 9. Jursinic P A. Angular dependence of dose sensitivity of surface diodes. Med Phys. 2009;36(6):2165-2171. doi:10.1118/1.3125644.

10. Li QL, Deng XW, Chen LX, Huang SM. The angular dependence of a 2 - dimensional diode array and the feasibility of its application in verifying the composite dose distribution of intensity - modulated radiation therapy. Chin J Cancer. 2010;29(6):617620. 
11. Li QL, Deng XW, Chen LX, Huang SM. The angular dependence of a 2 - dimensional diode array and the feasibility of its application in verifying the composite dose distribution of intensity - modulated radiation therapy. Chin J Cancer. 2010:617-620.

12. Jursinic P A, Sharma R, Reuter J. MapCHECK used for rotational IMRT measurements: step-and-shoot, TomoTherapy, RapidArc. Med Phys. 2010;37(6):2837-2846. doi:10.1118/1.3431994.

13. Brahme A. Correction for the angular dependence of a detector in electron and photon beams. 1985;24(October):301-304. doi:10.3109/02841868509134404.

14. Bouchard H, Kamio Y, Palmans H, Seuntjens J, Duane S. Detector dose response in megavoltage small photon beams. I. Theoretical concepts. Med Phys Med Phys. 2015;42(10):6033-6047. doi:10.1118/1.4930798.

15. Jin H, Keeling VP, Johnson DA, Ahmad S. Interplay effect of angular dependence and calibration field size of MapCHECK 2 on RapidArc quality assurance. J Appl Clin Med Phys. 2014;15(3):80-92.

16. Allgaier B, Schüle E, Würfel J. Dose reconstruction in the OCTAVIUS 4D phantom and in the patient without using dose information from the TPS. PTW D91320006/00. 2013;(October):0-7.

17. Li G, Zhang Y, Jiang X, et al. Evaluation of the ArcCHECK QA system for IMRT and VMAT verification. Phys Medica. 2013;29(3):295-303. doi:10.1016/j.ejmp.2012.04.005.

18. Wolfsberger LD, Wagar M, Nitsch P, Bhagwat MS, Zygmanski P. Angular dose dependency of MatriXX TM and its calibration. J Appl Clin Med Phys. 2010;11(1):241251.

19. Shimohigashi Y, Araki F, Tominaga H, et al. Angular dependence correction of MatriXX and its application to composite dose verification. J Appl Clin Med Phys. 2012;13(5):198-214.

20. Feygelman V, Forster K, Opp D, Nilsson G. Evaluation of a biplanar diode array dosimeter for quality assurance of step-and-shoot IMRT. J Appl Clin Med Phys. 2009;10(4):64-78. doi:10.1120/jacmp.v10i4.3080. 
21. Charles PH, Crowe SB, Kairn T, et al. The effect of very small air gaps on small field dosimetry. Phys Med Biol. 2012;57(21):6947-6960. doi:10.1088/0031-9155/57/21/6947.

22. Utitsarn K, Alrowaili ZA, Stansook N, et al. Optimisation of output factor measurements using the magic plate 512 silicon dosimeter array in small megavoltage photon fields. $J$ Phys Conf Ser. 2017;777:012022. doi:10.1088/1742-6596/365/1/011001.

23. Texas Instrument. 64 Channel Analog Front End for Digital X-Ray Detector AFE0064. 2009;(September). http://www.ti.com/lit/ds/slas672/slas672.pdf.

24. Fuduli I, Newall MK, Espinoza AA, et al. Multichannel Data Acquisition System comparison for Quality Assurance in external beam radiation therapy. Radiat Meas. 2014;71:338-341. doi:10.1016/j.radmeas.2014.05.016.

25. Devic S, Tomic N, Aldelaijan S, et al. Linearization of dose-response curve of the radiochromic film dosimetry system. Med Phys. 2012;39(8):4850-4857. doi:10.1118/1.4736800.

26. Papaconstadopoulos P, Hegyi G, Seuntjens J, Devic S. A protocol for EBT3 radiochromic film dosimetry using reflection scanning. Med Phys. 2014;41(12):122101. doi:10.1118/1.4901308.

27. Rikner G, Grusell E. Selective shielding of a p-Si detector for quality independence. Acta Radiol Oncol. 1984;24(1):65-69. doi:10.3109/02841868509134367.

28. Das IJ, Kahn FM. Backscatter dose perturbation at high atomic number interfaces in megavoltage photon beams. Med Phys. 1989;16(3):367-375.

29. Werner BL, Das IJ, Khan FM, Meigooni AS. Dose perturbations at interfaces in photon beams. Med Phys. 1987;14(4):585-595.

30. Bouchard H, Kamio Y, Palmans H, Seuntjens J, Duane S. Detector dose response in megavoltage small photon beams. II. Pencil beam perturbation effects. Med Phys Med Phys. 2015;42(10):6048-6061. doi:10.1118/1.4930798. 